\title{
Les versions francophones de vulgarisation de la Convention internationale des droits de l'enfant
}

\section{Cécile Desoutter}

DOI: http://dx.doi.org/10.7359/814-2017-deso

\section{RÉSUMÉ}

Depuis sa signature en 1989, et au fur et à mesure de sa ratification par 193 pays, la Convention internationale des droits de l'enfant (CIDE) a donné lieu, dans de nombreux pays, à des versions de vulgarisation visant des publics de différents âges, langues ou cultures. L'étude prend en considération 15 versions de vulgarisation en français, produites dans des pays francophones ou par des organisations internationales. L'objectif est de mettre au jour, d'une part, en quoi elles se distinguent de la version officielle, d'autre part, dans quelle mesure chacune d'elles est influencée par la culture du pays/continent dans lequel elle est produite.

Mots-clefs: CADBE, CIDE, droits enfants, textes de vulgarisation, version officieuse.

Les Etats parties s'engagent à faire largement connaître les principes et les dispositions de la présente Convention, par des moyens actifs et appropriés, aux adultes comme aux enfants.

CIDE (1989), art. 42

Par son article 42, la Convention internationale des droits de l'enfant (CIDE) instaure un droit spécifique à l'éducation aux droits de l'enfant. De fait, l'article prévoit expressément que la connaissance des principes et disposi- 
tions contenues dans la Convention soit transmise aux enfants mais aussi aux adultes. Il rend également les États parties responsables de la mise en œuvre de cette transmission par des moyens actifs et appropriés, dont ils sont par ailleurs tenus de rendre compte dans leurs rapports initiaux ou périodiques au Comité des Droits de l'Enfant, organe de contrôle de la bonne application de la Convention. Cet article met donc clairement en évidence que la simple existence d'une convention internationale ne suffit pas à faire que celle-ci devienne objet d'attention, non seulement de la part des gouvernements, mais aussi de la part de la société.

La production ou la diffusion de versions de la CIDE adaptées à des publics spécifiques fait partie des mesures adoptées pour faire connaître les droits de l'enfant. Dans leur rapport au Comité, certains pays francophones plurilingues signalent ainsi la traduction de la CIDE en langues locales: par exemple, le Burkina Faso (1998) l'a traduite dans les quatre principales langues nationales: mooré-dioula-fulfuldé-gulmacema, tandis que la Suisse a pris des dispositions, suite aux observations du Comité, pour proposer une version en romanche (ONU 2015). Mais, même lorsque la langue française ne fait pas obstacle, c'est souvent le langage juridique qui empêche la bonne compréhension du texte. En effet, la Convention peut représenter un discours difficile à saisir, y compris par les adultes. Pour la rendre accessible à tout public, il ne faut donc pas seulement la diffuser mais aussi la vulgariser, c'est-à-dire mettre ses contenus à la portée de non-spécialistes. Cette vulgarisation est d'autant plus nécessaire que la CIDE reste encore un texte peu connu. De fait, une vingtaine d'années après sa publication, seul un quart des jeunes de 10 à 18 ans en Belgique (CODE 2007) en avait connaissance et sa notoriété était sensiblement la même en France en 2010 chez les jeunes de 13 à 18 ans (UNICEF - France 2010). Par ailleurs, une enquête originale menée sur tout le territoire du Sénégal par des enfants auprès d'enfants (CONAF 2011) signale qu'un enfant sénégalais sur deux a entendu parlé de la CIDE mais que seul un sur huit considère qu'elle protège les enfants.

Nous présenterons des versions de vulgarisation de la CIDE produites dans différents pays francophones afin d'observer et d'analyser comment ces discours seconds mettent en scène l'enfant, les adultes et l'État et les relations qu'ils entretiennent mutuellement. Notre analyse se veut comparative et mettra l'accent sur certaines différences entre les versions vulgarisées et le texte officiel mais aussi entre les versions vulgarisées ellesmêmes. 


\section{LA VULGARISATION DE TEXTES JURIDIQUES}

Lobjectif de la vulgarisation des textes juridiques est la vulgarisation des droits qu'ils contiennent. Il s'agit d'une communication destinée à sensibiliser un public non spécialisé aux règles et principes du droit. De fait, elle vise à mobiliser les citoyens, d'une part pour qu'ils puissent exercer leurs droits, d'autre part pour qu'ils n'ignorent pas le droit dans leurs comportements. Le processus de vulgarisation est différent de celui de simplification dans la mesure où ce dernier se réfère en général aux diverses initiatives (Plain Language Movement dans les pays de Common Law; Simplification du langage juridique, dans les pays francophones...) qui, à partir des années 1990, ont eu pour effet de poser des diagnostics et de proposer des solutions pour parvenir à une communication juridique claire (Fernbach 1995). Dans ce sens, la simplification est réalisée en amont par le rédacteur juridique à travers le recours à des principes rédactionnels basés sur la lisibilité et l'intelligibilité des textes, tandis que le travail de vulgarisation est réalisé en aval par le vulgarisateur une fois que la norme est édictée (cf. le site de l'AVIJED) ${ }^{1}$. Ceci étant, ainsi qu'on le verra plus loin, un certain nombre de versions reformulées de la Convention s'intitulent "version simplifiée", tout en ayant été rédigées des années après le texte officiel et dans un but de vulgarisation. Signalons encore que la vulgarisation diffère également de la diffusion du droit, qui consiste à rendre les textes juridiques accessibles aux citoyens (cf. en France le site internet Legifrance) sans nécessairement leur fournir les instruments pour les comprendre.

Vulgariser un discours juridique implique non seulement de le reformuler mais aussi de le recontextualiser pour l'adapter aux besoins et attentes d'un nouveau public de destinataires et parfois à un nouveau support, impliquant l'introduction de divers systèmes sémiotiques (Gotti 2012). Une fois définis le public visé et le média de diffusion, la difficulté de la vulgarisation consiste à faire des choix sur l'information à transmettre et sur la formulation de celle-ci, compte tenu que les textes seconds produits, même lorsqu'ils se présentent sous la même forme que le texte original, n'ont aucune valeur juridique. Le principe de vulgarisation de textes fondamentaux sur les droits humains n'est pas en soi nouveau (cf. la Déclaration universelle des droits de l'bomme, la Convention européenne des droits de l'bomme, la Charte africaine des droits de l'bomme et des peuples...),

1 Association pour la Vulgarisation de l'Information Juridique et l'Éducation au Droit. 
toutefois la caractéristique de la CIDE, comme nous l'avons mentionné, est que la vulgarisation du texte-même est implicitement prévue dans l'un de ces articles.

\section{LE CORPUS}

Parmi les nombreuses versions de vulgarisation en français, nous en analysons ici quinze, que nous avons collectées sur la Toile avec le moteur de recherche Google. Nous avons choisi d'étudier exclusivement des textes présentant les principes et dispositions sous forme d'articles numérotés et sans commentaires, suivant l'ordre du texte original. De façon à pouvoir effectuer des comparaisons article par article, le recueil exclut les nombreuses versions proposant des regroupements par thèmes. Ce critère de sélection restrictif, mais nécessaire pour obtenir un corpus homogène, a permis de rassembler quinze versions différentes (voir Annexe 1), provenant de divers pays francophones (Canada, Belgique, France, Luxembourg, Sénégal et Suisse), du Conseil de l'Europe et du Réseau d'Information sur les Droits de l'Enfant (CRIN). Nous n'avons trouvé qu'un seul document originaire d'un pays africain (le Sénégal), sans doute en raison du fait qu'internet ne constitue pas encore un média de diffusion privilégié en Afrique, ce qui n'empêche pas que ces versions existent sur support papier. De fait, dans leur rapport au Comité des Droits de l'Enfant, certains pays (par exemple la RDC, le Cameroun, le Burkina Faso) signalent qu'ils ont élaboré des versions reformulées de la Convention, auxquelles il ne nous a toutefois pas été possible d'accéder. Ceci limite malheureusement l'analyse comparative culturelle. Cependant, à défaut de ces textes, nous tirerons parti de l'existence d'une Charte africaine des droits et du bien-être de l'enfant (CADBE), adoptée par les chefs d'État et de gouvernement de l'Organisation de l'Unité Africaine en juillet 1990, soit moins d'un an après la CIDE. L'objectif de la Charte est d'inclure une perspective africaine par rapport aux concepts des droits de l'enfant, complétant donc la Convention internationale. Notons à cet égard que selon l'enquête sénégalaise citée plus haut (CONAF 2011), plus de deux personnes interrogées sur trois (69\%), surtout au niveau communautaire, considèrent que les droits de l'enfant énoncés dans la CIDE sont contraires à leur culture. Lorsque que cela s'avérera utile pour mettre en évidence une éventuelle influence culturelle sur certains choix rédactionnels de la version de vulgarisation sénégalaise de la CIDE, nous tiendrons compte de la CADBE 
et y ferons référence, ainsi qu'à sa "version simplifiée" proposée par le $\mathrm{Bu}-$ reau Régional d'Afrique d'Amnesty International.

Il est possible de regrouper les textes du corpus en plusieurs catégories, à commencer par leurs émetteurs. On trouve ainsi des versions de vulgarisation provenant des organisations gouvernementales (UNICEF, Délégation aux Droits de l'Enfant, Conseil de l'Europe) ou non gouvernementales (COFRADE, Humanium, CRIN ...). Le même émetteur (en particulier l'UNICEF) peut être à la source de versions différentes, en fonction des publics ou des pays de diffusion. Par ailleurs, dans les pays plurilingues, un même texte peut être proposé dans les diverses langues nationales et en un nombre encore plus grand de langues sur les sites qu'on appellera ici 'internationaux', par exemple le Conseil de l'Europe et le Réseau International d'Information sur les Droits de l'Enfant (CRIN). S'il est relativement facile d'identifier l'émetteur d'une version, bien que celle-ci puisse été affichée sur les pages de divers sites qui n'en citent pas l'origine, il est en revanche pratiquement impossible de savoir qui a effectivement contribué à l'élaboration du texte. Ainsi, à part de rares exceptions, nous ignorons si les reformulations sont le résultat du travail d'une seule personne ou d'équipes pluridisciplinaires, qui impliquent ou pas la participation des destinataires (les enfants par exemple). De même, dans le cas de versions plurilingues, nous ignorons quelle a été la langue de départ.

Un autre regroupement des textes peut être fait sur la base de leur dénomination, qui renvoie soit à la forme - qu'il s'agisse de la longueur ou du langage utilisé - soit au destinataire. La référence à la longueur (version "résumée", "abrégée", "condensée") donne la tendance générale des versions du corpus qui ont en commun d'être plus brèves que le texte officiel et donc de découler d'une opération de sélection dans l'information à transmettre. En dehors du fait que les articles sont plus courts, la plupart du temps le préambule est absent tout comme les articles 43 à 54 concernant les dispositions relatives à l'application de la Convention. La référence au langage ("version simplifiée”, "en langage simple” ou "en langage clair et simple") concerne cinq versions et en particulier les trois en provenance du Canada, ce qui reflète la sensibilité canadienne à la question de la littératie. La référence au destinataire ("texte adapté aux enfants", "version pour les enfants", "kids"), présente dans cinq versions, peut permettre de distinguer au premier abord les textes qui sadressent expressément aux enfants de ceux destinés à un public moins défini.

Au-delà de la dénomination générale, huit versions sur quinze font précéder chaque article d'un titre annonçant le principe ou la disposition 
qu'il contient. Ces titres, qui ne figurent pas dans la version originale, permettent un accès plus immédiat à l'information et en facilitent la compréhension.

\section{QUEL ENFANT?}

\subsection{Le choix énonciatif}

Dans les versions de vulgarisation, l'enfant peut être à la fois celui dont on parle et celui à qui l'on parle. Ainsi, dans huit des quinze textes du corpus, l'énonciation est entièrement ou partiellement à la $2^{\mathrm{e}}$ personne du singulier et la présence de l'embrayeur tu permet de cataloguer sans aucun doute ces textes comme des versions pour enfants. Cela dit, tous les textes expressément destinés aux enfants ne s'adressent pas directement à eux, comme le montre la Version pour les enfants de War Child rédigée à la $3^{\mathrm{e}}$ personne. Comme on le constatera à partir des extraits ci-dessous, le choix de l'énonciation peut avoir une influence remarquable sur la réception du texte.

Comparons ainsi un extrait de l'article 7 de la version officielle de la CIDE relatif au droit qu'a l'enfant d'avoir un nom:

Art. 7 L'enfant est enregistré aussitôt sa naissance et a dès celle-ci le droit à un nom [...] (CIDE)

aux versions de vulgarisation dont l'énonciation est à la $3^{\mathrm{e}}$ personne:

Art. 7 Chaque enfant ${ }^{2}$ sera enregistré dès sa naissance et aura droit à un nom [...] (Suisse - Projuventute)

Art. 7 Tout enfant a le droit à un nom [...] (UNICEF - Canada)

Art. 7 Les enfants ont le droit d'avoir un nom [...] (War Child - NL/ Canada)

et à la $2^{\mathrm{e}}$ personne:

Art. 7 Tu as droit à l'enregistrement légal de ta naissance, à un nom $[\ldots]$ (CoE)

Art. 7 Dès ta naissance, tu as le droit d'avoir un nom (Humanium Suisse)

2 Notre soulignement. 
La règle de droit est caractérisée par l'exigence de généralité et par une grande distance entre l'émetteur et de destinataire. À cet égard, Cornu (1990) souligne que l'énonciation à la troisième personne du singulier dans le discours législatif, auquel nous associons ici le discours des conventions, est la plus apte à se dépouiller de personnalité et à exprimer l'unité générique. Dans ce contexte, les énoncés "l'enfant", "chaque enfant”, "tout enfant", "les enfants" présentent l'intérêt de désigner de manière absolue à la fois l'ensemble des enfants et chacun pris individuellement. Cependant, un enfant, lecteur de la Convention, pourrait ne pas ne s'identifier à cette non-personne benvenistienne. Le choix du $t u$, en revanche, permet une communication personnalisée, adressée non pas à un enfant générique mais à l'enfant-sujet singulier et unique, qui peut plus facilement se reconnaître comme le destinataire des droits énoncés dans la Convention.

À propos de l'effort de personnalisation de la communication destinée aux enfants, il est intéressant de noter que dans la version de vulgarisation de la Charte africaine, l'énonciation est à la première personne du pluriel. Ainsi, dans son article 6, qui correspond à l'article 7 de la CIDE, il est écrit:

Art. 6 Nous avons le droit dès notre naissance de porter un nom [...] (CADBE - Version vulgarisée par Amnesty International Afrique)

Ici, c'est l'enfant qui affirme son droit à porter un nom. La reformulation à la première personne déplace donc complètement le point de vue, dans la mesure où il ne s'agit plus de dire que l'enfant a des droits ( $3^{\mathrm{e}}$ pers.), ni à l'enfant qu'il a des droits ( $2^{\mathrm{e}}$ pers.) mais de lui faire dire qu'il a des droits (1're pers.). Par ailleurs, le recours au pluriel ne met plus l'enfant en position individuelle mais collective. Ce choix de ne pas mettre en scène un je mais un nous peut sans doute être relié à la notion de communauté qui prévaut en Afrique sur celle d'individualité.

\subsection{Le choix du genre grammatical}

En français, conformément au principe de généralité, le masculin grammatical désigne à la fois les personnes de sexe masculin et féminin. Toutefois, depuis longtemps déjà, en Suisse et au Canada, la sensibilité à la parité linguistique a amené à la pratique d'une rédaction administrative et législative épicène. Ceci laisse supposer que les versions de vulgarisation de la CIDE publiées dans ces pays sont susceptibles de tenir compte du genre biologique des enfants. Pour vérifier cette hypothèse, l'article 34.b 
de la Convention est approprié puisqu'il parle du droit à la protection contre l'exploitation sexuelle:

Art. 34.b [...] que des enfants ne soient exploités à des fins de prostitution ou autres pratiques sexuelles illégales (CIDE)

Dans les versions de vulgarisation, le genre grammatical peut donner lieu à trois types de formulation qui vont du masculin générique:

Art. $34[$ [... tu ne sois pas exploité à des fins de prostitution (COFRADE France)

à la neutralisation:

Art. 34 L'Etat s'engage à protéger les enfants contre toutes les formes d'exploitation et violence sexuelle [...] (UNICEF - Canada)

à la particularisation (ou féminisation) ${ }^{3}$ :

Art. $34[\ldots]$ tu ne sois pas exploité(e) à des fins de prostitution (Humanium - Suisse)

Art. $34[$ [...] tu ne sois pas incité(e) (qu’on te donne envie) ou contraint (que tu sois obligé(e)) à te livrer à une activité sexuelle illégale (Droitenfant - France)

Seules deux versions de vulgarisation recourent à l'usage du doublet masculin-féminin, dans tout ou partie de l'article 34 (voir ci-dessus Humanium et Droitsenfant), mettant grammaticalement en évidence que filles et garçons sont concernés. Dans le premier cas, celui de la version publiée par l'ONG suisse Humanium, ce choix ne surprend pas compte tenu que l'écriture épicène fait partie des pratiques de rédaction de la Confédération. Ce principe n'est cependant pas systématiquement appliqué puisque les deux autres versions de vulgarisation publiées en Suisse (UNICEF et Projuventute Canton de Vaud) suivent l'exemple du texte officiel de la CIDE et se limitent au masculin générique, tout comme les trois versions diffusées au Canada. Dans le cas de l'autre texte, publié sur le site http://www.droitsenfant.fr, l'option du recours aux doublets est plus difficile à expliquer dans la mesure où les pratiques rédactionnelles en France tendent à l'éviter, lui préférant l'usage du masculin générique, sauf exceptions (Desoutter 2013). Il faut noter que pour les deux versions en

3 Le terme de particularisation semble plus opportun lorsque l'objectif poursuivi est davantage de mettre les genres côte à côte que de faire apparaître systématiquement les femmes (cf. Abbou 2001). 
question, le choix de la particularisation du discours dans l'article 34 ne s'étend pas forcément aux autres articles ou à tous les mots susceptibles de prendre une forme double masculin-féminin. Ainsi, on relève les options suivantes:

- Humanium: exploité(e) (art. 34); soumis, condamné(e) (art. 37); accusé (art. 40.1); présumé(e) innocent(e) (art. 40.22).

- Droitsenfant: exploité(e), contraint (art. 34); soumis, exécuté (art. 37); accusé(e) (art. 40.1); présumé(e) innocent(e) (art. 40.2).

Le recours au doublet, là où il serait possible, n'est donc pas systématique. Toutefois, même épisodique, il révèle une attention particulière des rédacteurs (ou rédactrices!) à la question du genre grammatical et à la visibilité tant féminine que masculine. Puisque dans les deux cas, l'énonciation est à la deuxième personne, on peut considérer qu'elle accroît encore la personnalisation de la communication, permettant à l'enfant de se reconnaître comme destinataire en tant que fille ou garçon.

\section{L'ENFANT EN RELATION AVEC LES ADULTES}

La Convention des droits de l'enfant est un instrument ayant force obligatoire, par lequel les États parties s'engagent à prendre un certain nombre de mesures. Elle contient donc la liste complète des obligations positives que l'État déclare assumer envers les enfants: obligations directes et indirectes. L'article 5 est relatif à des obligations indirectes puisqu'il énonce que l'État doit veiller à permettre à d'autres que lui-même de remplir pleinement leur rôle et leurs responsabilités en matière de bien-être et de protection des enfants:

Art. 5 Les Etats parties respectent la responsabilité, le droit et le devoir qu'ont les parents ou, le cas échéant, les membres de la famille élargie ou de la communauté, comme prévu par la coutume locale, les tuteurs ou autres personnes légalement responsables de l'enfant, de donner à celui-ci, d'une manière qui corresponde au développement de ses capacités, l'orientation et les conseils appropriés à l'exercice des droits que lui reconnaît la présente Convention (CIDE - Version officielle)

Il est intéressant d'observer ce qui a été conservé dans l'opération de reformulation de cet article. Ainsi, alors que la version officielle donne 
aux parents et à leurs substituts - membres de la famille élargie ou de la communauté, tuteurs ou autres personnes légalement responsables - la responsabilité de guider l'enfant, aucune version de vulgarisation ne mentionne toutes ces entités. De fait, les parents figurent dans onze versions, la famille dans six versions, la famille élargie dans trois, la communauté dans une seule version et les personnes légalement responsables également. L'opération de réécriture tend ainsi à limiter les porteurs de la responsabilité à une cellule familiale étroite, ce qui ancre les versions de vulgarisation analysées dans une conception occidentale, alors que le texte original tendait plutôt à l'ouverture. À cet égard, il faut noter que seule la version de vulgarisation publiée au Sénégal fait référence à la communauté, tout en limitant cependant la présence du mot au seul titre, sans le reprendre ultérieurement dans l'article:

Art. 5 Les droits et devoirs des parents, des familles et de la communauté.

Les Etats doivent respecter le droit des parents et de la famille de prendre soin du développement de leur enfant (Sénégal)

La version sénégalaise est également la seule à utiliser l'adjectif possessif, leur enfant, mettant ainsi en évidence un lien d'appartenance qui n'apparait pas revanche dans les versions recourant à l'article défini, comme cidessous par exemple:

Art. 5 L'Etat s'engage à respecter les droits et responsabilités des parents pour ce qui est d'encadrer l'enfant d'une manière qui corresponde au développement de ses capacités (UNICEF - Canada)

Pour revenir au mot communauté, on le trouve également présent dans les versions de vulgarisation suisses plurilingues proposées par l'UNICEF et par Humanium dans l'art. 23.1 - concernant la participation des enfants handicapés à la vie de la communauté - et ce quelle que soit la langue de publication (comunidad, community, Gemeinshaft). Dans l'article correspondant de la version officielle en français, c'est en revanche le mot collectivité qui figure, alors que la version officielle en anglais recourt aussi au terme community. On relève ainsi, à travers les versions française et anglaise officielles de la CIDE, ce que Philippe d'Iribarne fait remarquer dans un tout autre contexte $(2009,26)$, à savoir "la réticence française à l'égard d'une appartenance communautaire" et, partant, la tendance à parler de collectivité, voire de société, là où les textes anglais parlent de communauté. Toujours à propos de l'article 23 , la version de vulgarisation diffusée au Sénégal, qui a sans doute pour base le texte officiel français, 
ne fait référence ni à la collectivité ni à la communauté mais à la société en énonçant le droit des enfants handicapés à "mener une vie sociale complète". Si l'on considère à présent l'article correspondant de la CADBE (art. 13), on relève à la fois l'encouragement "à participer activement à la vie communautaire" et "le droit à l'intégration sociale" ainsi qu'à "l'épanouissement individuel de l'enfant handicapé”. De façon générale, on peut constater que le mot communauté ou communautaire n'est présent que deux fois dans la version officielle de la CIDE (préambule et art. 5), alors qu'il l'est dix fois dans le texte officiel de la CADBE et onze fois dans la version de vulgarisation de cette dernière publiée par Amnesty International Afrique. On retrouve ici l'importance, déjà signalée plus haut, de la notion de communauté en Afrique, que celle-ci soit locale, nationale, internationale, religieuse ou d'information. Il apparait ainsi nettement qu'au-delà des réalités qu'il recouvre, le mot communauté a une forte charge culturelle et qu'il est connoté de façons différentes d'une langue à l'autre, mais aussi d'un pays (ou continent) à l'autre. C'est sans doute ce qui peut expliquer son apparition plus ou moins fréquente dans les textes issus de pays francophones.

Encore à propos de communauté, il faut relever une différence fondamentale entre la Convention et la Charte africaine. Ainsi, l'art. 31 de la CADBE est un peu l'autre face de l'art. 5 de la CIDE puisqu'il stipule que "tout enfant a des responsabilités envers sa famille, la société, l'Etat et toute autre communauté reconnue légalement ainsi qu'envers la communauté internationale [...]». Cet énoncé traduit la conception globalisante des droits de l'enfant (et de l'homme) en Afrique, qui prescrit l'équilibre entre droits et devoirs, entre individu et communauté (Boukongou 2006). En effet, comme le relève Grosjean (2007), l'appartenance de l'enfant à une communauté, à un groupe, justifie que s'applique, dans les cultures africaines, le principe communautaire de la réciprocité des droits et des obligations. Il n'est donc pas surprenant que l'article 31 de la version de la Charte africaine vulgarisée par Amnesty International Afrique reprent pratiquement dans son entier le texte de la version officielle, en énonçant envers qui l'enfant a des responsabilités et en insistant tout particulièrement sur la communauté internationale:

Art. 31 Tout enfant a des responsabilités envers sa famille, la société, l'Etat et même envers la communauté internationale [...] (CADBE Version de vulgarisation Amnesty International Afrique) 


\section{L'ENFANT EN RELATION AVEC L'ÉTAT}

\subsection{Le choix du désignateur}

Comme nous l'avons souligné précédemment, la majorité des articles de la Convention énonce les engagements pris par les États parties. Ceci explique que le mot État soit présent cent vingt et une fois dans le texte officiel en français. Dans les versions de vulgarisation, d'autres choix lexicaux peuvent être faits, comme l'illustre par exemple les diverses reformulations de l'article 4 de la CIDE:

Art. 4 Les Etats parties s'engagent à prendre toutes les mesures législatives, administratives et autres qui sont nécessaires pour mettre en œuvre les droits reconnus dans la présente Convention (CIDE Version officielle)

On relève que huit des versions de vulgarisation de cet article 4 reprennent le mot État, quatre d'entre elles introduisent gouvernement et trois le mot pays, selon les cas au singulier ou au pluriel. Pour l'ensemble des articles, on constate, sans grande surprise, que les deux versions en provenance de France sont celles qui recourent le plus souvent au mot Etat: cinquante deux occurrences pour le texte de la COFRADE, quarante quatre pour celui de Droitsenfant. En revanche, ce mot ne figure que trois fois dans la version de la Fédération Wallonie-Bruxelles et n'apparait jamais dans celle d'UNICEF - Belgique (dans l'une et dans l'autre on parle de pays). Compte tenu de la nature "discrète" de l'État belge, pour reprendre l'adjectif employé par Alain Touraine $(1973,260)$, ce choix reflète bien une tendance culturelle. Les versions publiées en Suisse, quant à elles, n'excluent pas le désignateur État; toutefois celle de l'UNICEF le privilégie (27 occurrences) par rapport aux versions des ONG Humanium (1 occurrence) et Projuventute du Canton de Vaud (5 occurrences), qui lui préfèrent très largement le mot pays.

Le choix n'est pas sans conséquence puisque État donne l'idée qu'un appareil politique, juridique et administratif structuré veille sur l'enfant. Pays, en revanche, réfère plus à un territoire ou à l'ensemble des habitants et des forces économiques et sociales d'une nation (Larousse en ligne), ce qui fait penser à un effet plus collectif, plus affectif, mais peut-être aussi plus abstrait de l'engagement. Quant à gouvernement, le désignateur le moins souvent utilisé, il pourrait donner une impression moins stable de la protection, susceptible de varier en fonction de la permanence et de l'orientation de l'équipe au pouvoir. 


\subsection{Genres discursifs et choix modaux}

En dehors de la désignation, l'analyse du corpus des versions vulgarisées permet de mettre en évidence un glissement de la notion d'État-détenteur d'obligations à celle, corollaire, d'enfant-objet des obligations de l'État, mais aussi à celle d'enfant-détenteur de droits. Ce glissement apparaît tout à fait clairement dans l'article 12 concernant le droit à la libre expression de l'enfant:

Art. 12 Les Etats parties garantissent à l'enfant qui est capable de discernement le droit d'exprimer librement son opinion sur toute question l'intéressant, les opinions de l'enfant étant dûment prises en considération eu égard à son âge et à son degré de maturité. A cette fin, on donnera notamment à l'enfant la possibilité d'être entendu dans toute procédure judiciaire ou administrative l'intéressant [...] (CIDE - Version officielle)

Dans presque toutes les versions de vulgarisation, le droit de l'enfant est exprimé en premier lieu, l'obligation de l'État passant au second plan:

Art. 12 Tu as droit à la liberté d'opinion. Dès que tu en es capable, tu as le droit de donner ton avis sur des choses qui se rapportent à toi et ton pays doit y faire attention (Fédération Wallonie-Bruxelles)

Art. 12 Dès que tu en es capable, tu as le droit de donner ton avis à propos de tout ce qui te concerne. Les Etats doivent te garantir ce droit (COFRADE - France)

Mais il arrive aussi que le rôle de l'État comme détenteur de l'obligation et garant des droits soit complètement omis dans l'opération de reformulation:

Art. 12 L'enfant a le droit d'exprimer son avis à propos de tout ce qui le ou la concerne et il convient d'accorder à cet avis tout le poids qu'il mérite (Projuventute - Suisse)

Art. 12 Les enfants ont le droit d'exprimer librement leur opinion, qui doit être prise en considération, sur les questions qui les touchent (UNICEF - Canada)

Dans la CIDE, les obligations de l'État envers les enfants sont en général exprimées par des verbes modaux, des performatifs ou des verbes au présent de l'indicatif, comme le montre l'extrait suivant de l'article 3 relatif à l'intérêt supérieur de l'enfant: 
Art. 3.1 L'intérêt supérieur doit être [modal déontique] une considération primordiale $[. .$.

Art. 3.2 Les Etat s'engagent à [...] [performatif], prennent les mesures [présent déontique ou constatif]

Art. 3.3 Les Etats veillent à [présent déontique ou constatif] ce que le fonctionnement des services [...] soit conforme (CIDE - Version officielle)

Dans les versions de vulgarisation, les verbes performatifs et le présent de l'indicatif tendent à disparaître et lorsqu'une obligation est exprimée, elle est presque systématiquement formulée au moyen du déontique devoir:

Art. 3 Pour toutes les décisions qui te concernent, ton intérêt doit être pris en compte $[. .$.

Les pays doivent te protéger [...]

Les pays doivent veiller à ce que [...] (Humanium - Suisse)

Art. 3 Toute action ou décision concernant les enfants doit tenir compte de ce qui est le mieux pour toi ou pour les enfants en général (CoE)

Art. 3 Tous les adultes des institutions publiques, des tribunaux et des administrations doivent faire ce qui est le mieux pour toi. Quand ils prennent des décisions, ils doivent penser à la façon dont elles vont affecter les enfants (ORK - Luxembourg)

Selon une convention de langage, en matière juridique, l'indicatif présent suffit non seulement à marquer le droit mais aussi, plus spécifiquement, à marquer l'obligation. Le guide de légistique publié en France sur le site Légifrance énonce ainsi qu'“Il n'y a pas lieu, sous prétexte de renforcer le caractère impératif d'une obligation, de recourir au mot 'doit'; le simple présent du verbe principal suffit”. En Suisse, la Chancellerie Fédéral indique également dans son guide linguistique en ligne que l'indicatif est "le temps par excellence du discours législatif" avec pour justification que "plus c'est simple mieux c'est, et plus c'est simple plus le style est caractéristique du droit". Le présent de l'indicatif participe donc du mouvement de simplification du droit et du langage juridique. Suivant ce raisonnement, on peut considérer que, dans le texte officiel de la CIDE, l'usage modéré de l'auxiliaire modal devoir ou de ses équivalents sert simplement à rappeler la lecture prescriptive qu'il convient de faire du présent de l'indicatif.

Mais, au-delà de la simplification du langage juridique, la question de l'indicatif présent relève de la philosophie du droit et oppose deux concep- 
tions. D'une part, la théorie impérativiste répute que le présent de l'indicatif a valeur impérative, parce que l'énoncé juridique est normatif, quelle que soit sa forme grammaticale (Kalinowski 1972); d'autre part la théorie descriptiviste considère que "ce mode correspond à la fonction propre du droit" qui est "de fixer la condition de chacun" (Villey 1974, 39). Cornu (1990), partisan d'une conception plus descriptiviste qu'impérativiste de la règle de droit, précise à cet égard que l'indicatif présent offre l'avantage de commander de façon plus douce, plus discrète. Selon lui, ce mode pourrait même faire imaginer que la règle énoncée n'est pas arbitrairement imposée, mais naturellement fondée et qu'en conséquence le droit est proche de la nature des choses. Exprimer la norme par une phrase à l'indicatif (entendue comme non explicitement modalisée) crée un "effet de réalité" qui favoriserait le respect de la règle elle-même. Si l'on suit son raisonnement, en énonçant par exemple que "les Etats veillent" (CIDE), on anticipe la réalisation, on montre le sujet agissant, conformément à la règle.

En revanche, dans les versions de vulgarisation, les énoncés "les pays doivent veiller" (Humanium - Suisse), “doivent tenir compte” (ORK) procèdent d'un autre raisonnement et mettent l'accent sur le fait que le présent de l'indicatif renverrait plutôt à des procès désactualisés, à la description d'un événement prototypique idéal, à une situation exemplaire seulement potentiellement effective, sans ancrage historique (Gerbe 2006) et qui ne garantit finalement rien. La présence fréquente de l'auxiliaire modal dans ces discours a par conséquent pour fonction de rappeler le caractère obligatoire des dispositions, peut-être aussi rendu nécessaire par le fait que le texte de vulgarisation, compte tenu de sa nature générique, n'acquiert pas une valeur injonctive à la différence du texte de la Convention.

On remarquera aussi que, démuni d'énoncés performatifs-constitutifs dans lesquels “l'Etat s'engage à”, “veille à”, le texte reformulé s'éloigne des caractéristiques de la Convention pour ressembler plus à une déclaration. Or, une déclaration a un poids moral parce qu'elle est adoptée par la communauté internationale, mais elle n'a aucune valeur contraignante. C'est précisément à cause de cette absence d'effet contraignant de la Déclaration des droits de l'enfant (1959) que la Convention des droits de l'enfant a été adoptée en 1989 puis ratifiée par 193 pays sur 195. Les versions vulgarisées, dont les énoncés n'ont pas valeur performative, tendent donc souvent par leur formulation à se rapprocher plus de la Déclaration que de la Convention, ainsi qu'on peut l'observer ci-dessous à partir du droit à l'éducation qui est présent à la fois dans le texte de 1959 et dans celui de 1989: 
Art. 28 Les Etats parties reconnaissent le droit des enfants à l'éducation et en particulier, en vue d'assurer l'exercice de ce droit progressivement et sur la base de l'égalité des chances:

a) Ils rendent l'enseignement primaire obligatoire et gratuit pour tous; [...] (CIDE - Version officielle 1989)

Princ. 7 L'enfant a droit à une éducation qui doit être gratuite et obligatoire au moins aux niveaux élémentaires [...] (Déclaration 1959)

Art. 28. Tu as droit à l'éducation. La discipline scolaire doit respecter ta dignité en tant qu'être humain. L'enseignement primaire doit être gratuit et obligatoire $[. .].(\mathrm{CoE})$

Art. 28 L'enfant a droit à l'éducation. L'enseignement primaire doit être obligatoire et gratuitement accessible pour tous (Projuventute Suisse)

\section{CONCLUSION}

Les versions de vulgarisation de la CIDE examinées mettent en scène un enfant qui, selon les cas, se positionne sur un continuum: de l'individu abstrait et générique, dont parle le discours, à l'individu concret et unique, auquel sadresse le discours. Les adultes responsables de lui sont plus souvent présentés comme membres d'une cellule familiale nucléaire que d'une communauté élargie. Quant aux États parties, qu'ils soient désignés comme État, pays, ou gouvernement, ils n'apparaissent qu'au second plan et sont plus ou moins présents selon les émetteurs des textes. Le fait d'avoir pu recueillir des versions émanant de pays différents permet d'interpréter que certaines dissemblances entre les unes et les autres résultent de l'influence des cultures nationales. Cependant, la principale différence se situe plutôt entre l'ensemble des versions de vulgarisation et le texte officiel: toutes mettent l'accent sur les droits des enfants plutôt que sur les obligations des États. Il n'y a pas lieu de s'en étonner puisque les textes analysés sont des discours seconds, recontextualisés, qui ont pour objectif celui énoncé dans l'article 42 du discours premier: "faire largement connaître les principes et les dispositions de la [...] Convention”. 
ANNEXE:

VERSIONS DE VULGARISATION

\begin{tabular}{|c|c|c|c|c|c|}
\hline & DÉNOMINATION & ORGANISMES & OG/ONG & Pays & LANGUES \\
\hline 1 & Droits de l'enfant & $\begin{array}{l}\text { UNICEF - Belgique } \\
\text { Kids }\end{array}$ & OG & Belgique & $\begin{array}{l}\text { français } \\
\text { néerlandais }\end{array}$ \\
\hline 2 & $\begin{array}{l}\text { Convention } \\
\text { internationale des droits } \\
\text { de l'enfant }\end{array}$ & $\begin{array}{l}\text { Fédération Wallonie- } \\
\text { Bruxelles } \\
\text { Délégué Général } \\
\text { aux Droits de l'Enfant }\end{array}$ & OG & Belgique & français \\
\hline 3 & $\begin{array}{l}\text { La Convention } \\
\text { en résumé }\end{array}$ & $\begin{array}{l}\text { ORK-Ombuds - Comité } \\
\text { fir d'Rechter vum Kand } \\
\text { (Ombuds Comité } \\
\text { pour les Droits } \\
\text { des Enfants) }\end{array}$ & OG & Luxembourg & français \\
\hline 4 & $\begin{array}{l}\text { Convention relativ } \\
\text { aux droits de l'enfant } \\
\text { des Nations Unies } \\
\text { en langage clair et simple }\end{array}$ & $\begin{array}{l}\text { UNICEF - Canada / } \\
\text { Réseau Ontarien } \\
\text { d'Éducation Juridique }\end{array}$ & OG & Canada & $\begin{array}{l}\text { français } \\
\text { anglais }\end{array}$ \\
\hline 5 & $\begin{array}{l}\text { Convention relative } \\
\text { aux droits de l'enfant } \\
\text { en langage clair }\end{array}$ & $\begin{array}{l}\text { EQUITAS } \\
\text { Centre International } \\
\text { d'Éducation aux Droits } \\
\text { Humains (se réfère } \\
\text { à une version produite } \\
\text { par l'UNICEF) }\end{array}$ & OG & Canada & $\begin{array}{l}\text { français } \\
\text { anglais }\end{array}$ \\
\hline 6 & $\begin{array}{l}\text { Convention relative } \\
\text { aux droits de l'enfant- } \\
\text { Version simplifiée }\end{array}$ & $\begin{array}{l}\text { Commission } \\
\text { des Droits } \\
\text { de la Personne } \\
\text { et des Droits } \\
\text { de la Jeunesse } \\
\text { du Québec }\end{array}$ & OG & Canada & français \\
\hline 7 & $\begin{array}{l}\text { Convention de l'ONU } \\
\text { relative aux droits } \\
\text { de l'enfant- } \\
\text { Version condensée }\end{array}$ & $\begin{array}{l}\text { Comité Suisse } \\
\text { pour l'UNICEF }\end{array}$ & OG & Suisse & $\begin{array}{l}\text { français } \\
\text { italien } \\
\text { allemand }\end{array}$ \\
\hline 8 & $\begin{array}{l}\text { Convention } \\
\text { des Nations Unies } \\
\text { des droits de l'enfant- } \\
\text { Version abrégée }\end{array}$ & $\begin{array}{l}\text { Projuventute Canton } \\
\text { de Vaud }\end{array}$ & ONG & Suisse & $\begin{array}{l}\text { Version } \\
\text { française } \\
\text { traduite } \\
\text { de la version } \\
\text { italienne } \\
\text { (Fondazione } \\
\text { Lelio Basso) }\end{array}$ \\
\hline 9 & $\begin{array}{l}\text { Convention } \\
\text { internationale } \\
\text { des droits } \\
\text { de l'enfant - Texte adapté } \\
\text { aux enfants dès } 10 \text { ans }\end{array}$ & Humanium & ONG & $\begin{array}{l}\text { Suisse } \\
\text { France } \\
\text { Allemagne }\end{array}$ & $\begin{array}{l}\text { français } \\
\text { anglais } \\
\text { allemand } \\
\text { espagnol }\end{array}$ \\
\hline
\end{tabular}




\begin{tabular}{|c|c|c|c|c|c|}
\hline & DÉNOMINATION & ORGANISMES & OG/ONG & PAYS & LANGUES \\
\hline 10 & $\begin{array}{l}\text { Convention des droits } \\
\text { de l'enfant - } \\
\text { Texte simplifié }\end{array}$ & $\begin{array}{l}\text { COFRADE } \\
\text { (Conseil Français } \\
\text { des Associations pour } \\
\text { les Droits de l'Enfant) } \\
\text { en collaboration avec le } \\
\text { Comité Français } \\
\text { pour l'UNICEF à } \\
\text { partir d'une première } \\
\text { version rédigée par la } \\
\text { branche belge de l'École } \\
\text { Instrument de Paix } \\
\text { (EIP) }\end{array}$ & ONG & France & français \\
\hline 11 & $\begin{array}{l}\text { Convention } \\
\text { internationale - } \\
\text { Texte adapté } \\
\text { aux enfants }\end{array}$ & $\begin{array}{l}\text { Texte annoté } \\
\text { par J.C. Champagnat } \\
\text { pour le site http:// } \\
\text { www.Droitsenfant. } \\
\text { fr, inspiré du texte de } \\
\text { l'École Instrument de } \\
\text { Paix (EIP), et souvent } \\
\text { repris par d'autres sites } \\
\text { français }\end{array}$ & & France & français \\
\hline 12 & $\begin{array}{l}\text { Version simplifiée } \\
\text { de la Convention relative } \\
\text { aux droits de l'enfant }\end{array}$ & $\begin{array}{l}\text { Réseau d'Apprentissage } \\
\text { et d'Échange } \\
\text { d'Informations } \\
\text { des Organisations } \\
\text { des Droits Humains } \\
\text { du Sénégal }\end{array}$ & ONG & Sénégal & \\
\hline 13 & $\begin{array}{l}\text { Convention } \\
\text { sur les droits de l'enfant- } \\
\text { Version pour les enfants }\end{array}$ & $\begin{array}{l}\text { War Child Holland } \\
\text { (dossier Big-Deal Droits } \\
\text { et Responsabilités) }\end{array}$ & ONG & $\begin{array}{l}\text { Hollande } \\
\text { Canada }\end{array}$ & $\begin{array}{l}\text { français } \\
\text { anglais }\end{array}$ \\
\hline 14 & $\begin{array}{l}\text { Convention des droits } \\
\text { de l'enfant - } \\
\text { Version pour les enfants }\end{array}$ & $\begin{array}{l}\text { Conseil de l'Europe } \\
\text { Direction de la Jeunesse } \\
\text { et du Sport (dossier } \\
\text { Repères Juniors) }\end{array}$ & OG & & $\begin{array}{l}\text { français } \\
\text { (35 langues - } \\
\text { Original } \\
\text { en anglais) }\end{array}$ \\
\hline 15 & $\begin{array}{l}\text { Texte de la Convention - } \\
\text { Résumé non officiel }\end{array}$ & $\begin{array}{l}\text { CRIN (Child } \\
\text { Rights International } \\
\text { Network) - Réseau } \\
\text { International } \\
\text { d'Information } \\
\text { sur les Droits } \\
\text { de l'Enfant }\end{array}$ & ONG & & $\begin{array}{l}\text { anglais } \\
\text { français } \\
\text { russe } \\
\text { espagnol } \\
\text { arabe }\end{array}$ \\
\hline
\end{tabular}




\section{RÉFÉRENCES BIBLIOGRAPHIQUES}

Abbou, Julie. 2001. L'Antisexisme linguistique dans les brocbures libertaires. Pratiques d'écriture et métadiscours. Thèse de Doctorat en Sciences du Langage, Université d'Aix-Marseille.

Boukongou, Jean-Didier. 2006. "Le système africain des droits de l'enfant". Cabiers de la recherche sur les droits fondamentaux 6: 97-108.

CODE. 2007. L'éducation aux droits de l'enfant à l'école. La situation en Communauté française. Bruxelles: Coordination des ONG pour les droits de l'enfant. [12/05/2016]. http://lacode.be/IMG/pdf/etude.pdf.

CONAF (Coalition Nationale des Associations et ONG en Faveur de l'Enfance). 2001. Droit de l'enfant au Sénégal. De la Convention à la réalité. CONAF Sénégal - Save the Children. [01/06/2016]. http://resourcecentre. savethechildren.se/sites/default/files/documents/5524_1.pdf.

Cornu, Gérard. 1990. Linguistique juridique. Paris: Montchrestien.

Desoutter, Cécile. 2013. "La prise en compte linguistique des femmes dans les discours électoraux. Une étude sur corpus". Dans Les corpus dans la communication spécialisée, dirigé par Cécile Desoutter, Dorothee Heller, et Michele Sala, 435-464. Bergamo: CELSB (Cerlis Series). [12/05/2016]. http://dinamico. unibg.it/cerlis/public/CERLIS_SERIES_4_16.Desoutter.pdf.

d'Iribarne, Philippe. 2009. "Entre français et anglais. Une entreprise se met en scène". Langage et société 129: 101-118.

Fernbach, Nicole. 1995. "La simplification du texte juridique. Une étude comparative". Dans Français juridique et science du droit, dirigé par Gérard Snow et Gérard Vanderlinden, 105-122. Bruxelles: Bruylant.

Gerbe, Rose-Marie. 2006. "Le présent de l'indicatif dans le discours juridique français". Dans Legal Language and the Search for Clarity, dirigé par Anne Wagner et Sophie Cacciaguidi-Fahy, 266-302. Berne: Peter Lang.

Gotti, Maurizio. 2012. "La riscrittura del testo divulgativo". Altre modernità 11: 145159. [12/05/2016]. http://riviste.unimi.it/index.php/AMonline/article/ view/2468.

Grosjean, Kathleen. 2007. "Les droits de l'enfant dans la Convention internationale relative aux droits de l'enfant et la Charte africaine des droits et du bienêtre de l'enfant. Similarités et différences". Nouvelle Tribune Internationale des droits de l'enfant 12: 7-13.

Kalinowski, Georges. 1972. La logique des normes. Paris: PUF.

ONU. 2015. Observations finales concernant les deuxième à quatrième rapports périodiques de la Suisse, soumis en un seul document. Comité des Droits de l'Enfant (CRC/C/CHE/CO/2-4).

Touraine, Alain. 1973. Production de la société. Paris: Seuil. 
UNICEF - France. 2010. La perception des adolescents sur leur situation et celle des droits de l'enfant en France. Paris. https://www.unicef.fr/userfiles/TNS_Sofres_ Synthese_Ados_et_droits_enfants.pdf.

Villey, Michel. 1974. "De l'indicatif dans le droit". Archives de philosophie du droit 19: 33-61.

\section{Sitographie}

AVIJED. [20/05/2016]. http://www.avijed.org/.

Chancellerie Fédérale - Services linguistiques centraux de la section française de la Chancellerie Fédérale Suisse. Guide linguistique des lois et ordonnances de la Confédération. [12/05/2016]. https://www.bk.admin.ch/dokumentation/ publikationen/00292/01597/index.html?lang=fr.

Dictionnaire Larousse. [20/05/2016]. http://www.larousse.fr/dictionnaires/francais. Légifrance. [12/05/2016]. http://www.legifrance.gouv.fr. 